\title{
Maryam Mirzakhani (1977-2017): Her Work and Legacy
}

\author{
Valentin Zagrebnov (Aix-Marseille Université, Marseille, France), Editor-in-Chief of the EMS Newsletter
}

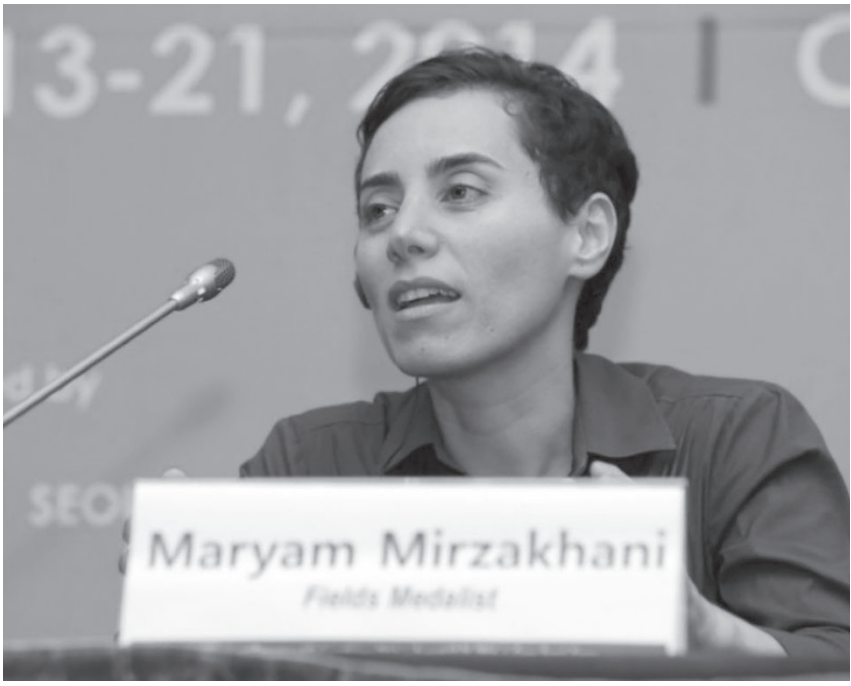

International Congress of Mathematicians, Seoul 2014. (Credit ?)

In 2014, Maryam Mirzakhani, a Harvard-educated mathematician and professor at Stanford University in California, was one of four Fields Medal winners announced by the International Congress of Mathematicians (ICM) at its conference in Seoul.

The award recognised Mirzakhani's sophisticated and highly original contributions to the fields of geometry and dynamical systems, particularly in understanding the symmetry of curved surfaces such as spheres.

Maryam Mirzakhani has made striking and highly original contributions to geometry and dynamical systems. Her work on Riemann surfaces and their moduli spaces bridges several mathematical disciplines - hyperbolic geometry, complex analysis, topology and dynamics - and influences them all in return. She gained widespread recognition for her early results in hyperbolic geometry and her most recent work constitutes a major advance in dynamical systems.

"This is a great honour. I will be happy if it encourages young female scientists and mathematicians," Maryam said. "I am sure there will be many more women winning this kind of award in coming years."

Born on 3 May 1977 and raised in Tehran, Maryam Mirzakhani initially dreamed of becoming a writer but, by the time she started high school, her affinity for solving mathematical problems and working on proofs had shifted her sights. "It is fun - it's like solving a puzzle or connecting the dots in a detective case," she said when she won the Fields Medal. "I felt that this was something I could do, and I wanted to pursue this path." Maryam said she enjoyed pure mathematics because of the elegance and longevity of the questions she studied.
"It is like being lost in a jungle and trying to use all the knowledge that you can gather to come up with some new tricks and, with some luck, you might find a way out," she added.

Maryam Mirzakhani became known on the international mathematics scene as a teenager, winning gold medals at both the 1994 and 1995 International Mathematical Olympiads - finishing with a perfect score in the latter competition.

She also won the 2009 Blumenthal Award for the Advancement of Research in Pure Mathematics and the 2013 Satter Prize of the American Mathematical Society.

In 2008, she became a professor of mathematics at Stanford, where she lived with her Czech husband and her daughter born in 2011.

In recent years, Maryam Mirzakhani has explored other aspects of the geometry of moduli space. Nonclosed geodesics in moduli space are very erratic and even pathological and it is hard to obtain any understanding of their structure and how they change when perturbed slightly. However, Maryam and co-authors have proved that complex geodesics and their closures in moduli space are in fact surprisingly regular, rather than irregular or fractal. It turns out that while complex geodesics are transcendental objects defined in terms of analysis and differential geometry, their closures are algebraic objects defined in terms of polynomials and therefore have certain rigidity properties.

Because of its complexities and inhomogeneity, moduli space has often seemed impossible to work on directly, but not to Maryam. She had a strong geometric intuition that allowed her to grapple directly with the geometry of moduli space. Fluent in a remarkably diverse range of mathematical techniques and disparate mathematical cultures, she embodied a rare combination of superb technical ability, bold ambition, far-reaching vision and deep curiosity.

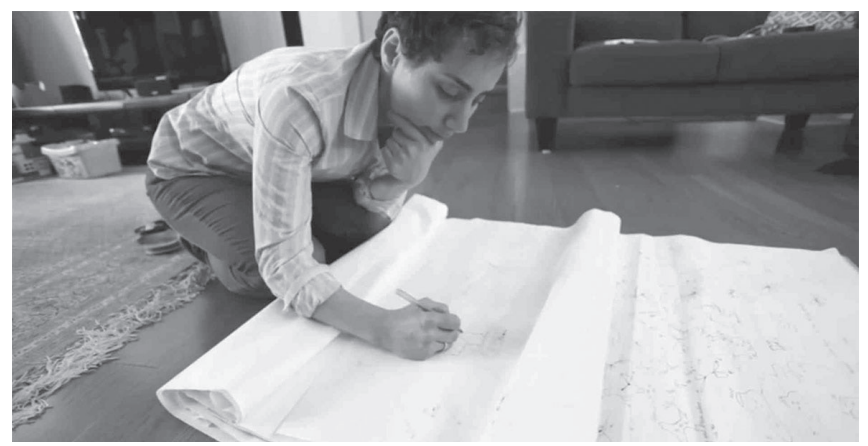

To solve problems, Maryam would draw doodles on sheets of paper and write mathematical formulas around the drawings. (Credit ?) 
Maryam Mirzakhani, the first woman to win the coveted Fields Medal, died on 15 July 2017 in a Californian hospital after a battle with cancer. She was only 40 .

Maryam's friend Firouz Naderi announced her death on Saturday 15 July on Instagram.
"A light was turned off today. It breaks my heart ... gone far too soon," wrote Naderi, a former director of Solar Systems Exploration at NASA.

"A genius? Yes. But also a daughter, a mother and a wife," he added in a subsequent post. 